\title{
Mesures des indicateurs de prolifération des larves de moustiques au niveau des mares permanentes et semi permanentes de Saga, Niger
}

\author{
Souleymane MAHAMANE IRO ${ }^{1 *}$, Yaro ALPHA SEYDOU² et Ali DOUMMA ${ }^{1}$ \\ ${ }^{1}$ Département de Biologie, Faculté des Sciences et Techniques, Université Abdou Moumouni de Niamey, BP \\ 10662 Niamey, Niger. \\ ${ }^{2}$ Université des Sciences, des Techniques et des Technologies- Facultés des Sciences et Techniques Bamako- \\ Mali, BP: E 3206. \\ "Auteur correspondant ; E-mail : irosouleymane@yahoo.fr
}

\section{RESUME}

Les Culicidae, communément appelés moustiques, par leur large distribution et leur abondance, sont impliqués dans la transmission de nombreuses maladies humaines et animales. La lutte contre ces insectes nécessite la connaissance de leurs lieux de développement dans lequel évoluent les stades pré imaginaux. La présente étude vise à caractériser ces gîtes larvaires en vue d'identifier les indicateurs entomologiques et écologiques de production de larves dans ces milieux. Pour ce faire, le $\mathrm{pH}$, la température, la conductivité, la teneur en oxygène dissout et la densité larvaire des culicidae ont été estimés à la suite des prospections des gîtes larvaires effectuées de juillet 2007 à mars 2018 à Saga, un quartier périurbain de la Ville de Niamey. Au total, 17 gîtes ont été identifiés dont cinq (5) gîtes permanents (29,41\%). Les surfaces d'eau artificielles ont été plus abondantes $(64,71 \%)$. L'étude sur la caractérisation des gîtes par la méthode ACP (Analyse par Composantes Principales) a permis de grouper les gîtes en trois (3) catégories: le groupe 1 constitué par des gîtes qui présentent un $\mathrm{pH}$ légèrement acide $(6,075$ à 7,035$)$ et un taux d'oxygène dissout élevé $(1,11$ à 2,32$)$. Le groupe 2 qui est constitué par des gites qui présentent une conductivité plus élevée (compris entre 0,97 à 1,08) ainsi qu'un pH légèrement alcalin $(7,51$ à 7,83$)$. Quant aux gîtes du groupe 3 , ils appartiennent en effet, à une zone de transition ou les trois (3) paramètres sont élevés à savoir le $\mathrm{pH}(8,02$ à 8,36$)$, le taux d'oxygène dissout $(1,43 \mathrm{mg} / \mathrm{l}$ à 2,54 $\mathrm{mg} / \mathrm{l})$ et de la conductivité $(1170 \mu \mathrm{S} / \mathrm{cm}$ à $1370 \mu \mathrm{S} / \mathrm{cm})$. L'étude a mis en évidence des affinités entre les espèces de moustiques et les conditions physico-chimiques des gîtes larvaires. C'est ainsi que An. gambiae s.l abonde dans les gîtes caractérisés par une température moins élevée, un $\mathrm{pH}$ neutre un taux d'oxygène dissout élevé alors que Culex pipens présente une affinité aux gîtes à forte conductivité et à taux d'oxygène dissout faible. (C) 2020 International Formulae Group. All rights reserved.

Mots clès : Culicidae, Paramètres physico-chimiques, gîtes larvaires, Saga, Niger.

\section{Measurements of mosquito larvae proliferation indicators in the permanent and semi-permanent ponds of Saga, Niger}

\begin{abstract}
Culicidae, commonly known as mosquitoes, by their wide distribution and abundance, are involved in the transmission of many human and animal diseases. The fight against these insects requires knowledge of their places of development in which the pre-imaginary stages evolve. The present study aims to characterize these
\end{abstract}


breeding sites in order to identify entomological and ecological indicators of larval production in these environments. To do this, the $\mathrm{pH}$, temperature, conductivity, dissolved oxygen content and larval density of culicidae were estimated following surveys of the breeding sites carried out from July 2007 to March 2018 in Saga, a peri-urban area of the City of Niamey. In total, 17 lodgings have been identified, including five (5) permanent lodgings $(29.41 \%)$. Artificial water surfaces were more abundant $(64.71 \%)$. The study on the characterization of the deposits by the ACP method (Principal Component Analysis) made it possible to group the deposits in three (3) categories: group 1 consisting of deposits that have a slightly acidic $\mathrm{pH}$ (6.075 to 7.035) and a high dissolved oxygen level (1.11 to 2.32). Group 2 which consists of gites which have a higher conductivity ( 0.97 to 1.08$)$ as well as a slightly alkaline $\mathrm{pH}$ (7.51 to 7.83). As for the group 3 deposits, they indeed belong to a transition zone where the three (3) parameters are high, namely the $\mathrm{pH}$ (8.02 to 8.36), the dissolved oxygen level $(1.43 \mathrm{mg} / 1$ to $2.54 \mathrm{mg} / \mathrm{l})$ and conductivity $(1170 \mu \mathrm{S} / \mathrm{cm}$ to $1370 \mu \mathrm{S} / \mathrm{cm})$. The study highlighted affinities between mosquito species and the physicochemical conditions of the breeding sites. This is how An. Gambiae sl abounds in deposits characterized by a lower temperature, a neutral $\mathrm{pH}$ a high dissolved oxygen level while Culex pipens has an affinity for deposits with high conductivity and low dissolved oxygen rate.

(C) 2020 International Formulae Group. All rights reserved.

Keywords: Culicidae, Physico-chemical parameters, breeding sites, Saga, Niger.

\section{INTRODUCTION}

Les Culicidae, communément appelés moustiques, par leur large distribution et leur abondance, sont impliqués dans la transmission de nombreuses maladies humaines et animales, d'où l'importance économique et l'intérêt médical accordés à ce groupe (Who, 2016).

En effet, plusieurs espèces de ces Culicidae, appartenant notamment aux genres Aèdes, Culex, ou Anophèles, sont des vecteurs d'organismes pathogènes (virus ou parasites) responsables de plusieurs affections telles que les arboviroses, les filarioses et le paludisme d'incidence médico-vétérinaire considérable (WHO, 2010). Selon Walter Reed Biosystematics Unit, (2001), les espèces les plus nombreuses se rencontrent sous les tropiques et à l'équateur, car beaucoup d'entre elles supportent mal les écarts thermiques. Les mâles et les femelles se nourrissent de sucs végétaux, mais seules les femelles se gorgent de sang.

Parmi les maladies transmises par ces moustiques, le paludisme est de loin celui qui constitue un problème de santé publique dans de nombreux pays tropicaux où près de 2 milliards de personnes sont exposées. Annuellement, l'incidence de la maladie dans le monde est estimée entre 300 et 500 millions de cas cliniques. En 2017, près de 219 millions de cas de paludisme ont été enregistrés dans le monde avec 435000 décès (OMS, 2018).

$\mathrm{Au}$ Niger comme dans la plupart des pays africains au Sud du Sahara, le paludisme est l'endémie majeure et la première cause de morbidité et de mortalité dans les groupes vulnérables ou elle provoque jusqu'à 3199064 cas présumés avec 2825329 cas confirmés pour un taux de létalité de $1.94 \%$ (OMS, Rapport annuel 2018).

Plusieurs espèces de moustiques vecteurs d'agents pathogènes ont été recensées au Niger (Hadiza $S$ et al. 2016). Ces espèces exploitent presque tous les types de collections d'eau pour pondre leurs œufs (Mourou et al. 2012).

D'une manière générale, les vecteurs évoluent dans des systèmes écologiques naturels et anthropisés (Makanga, 2016). C'est ainsi que dans les grandes agglomérations comme Niamey, capitale du Niger avec une urbanisation galopante et désordonnée associée aux nouvelles modes de vie de la population conduit à une augmentation des gîtes larvaires des moustiques et particulièrement ceux d'Anophèles faisant d'elles un fort réservoir de vecteurs potentiels. Ces nouvelles conditions favorables au développement larvaire et à la prolifération des moustiques sont à la base d'une forte augmentation du paludisme urbain 
constatée ces dernières années (Ahadji-Dabla et al., 2014, Morou et al., 2016).

A cet effet, pour rendre efficace les stratégies de lutte anti vectorielle contre ces Culicidae, une étude écologique préalable s'impose. De ce fait, la connaissance des gites larvaires dans lequel évoluent les stades préimaginaux des variations spatio-temporelles du développement et des activités des peuplements culicidiens est nécessaire (Abagli et al., 2014).

Or l'installation de fortes populations Culicidienne en milieu aquatique dépend essentiellement des caractéristiques physicochimiques de l'eau. En effet, ces milieux aquatiques, indispensables au développement des stades pré-imaginaux (œufs, larve et nymphe) ont des caractéristiques très variables et ne sont pas stables ni dans le temps ni dans l'espace. Herrel et al. (2001) rapportent que les paramètres physico-chimiques jouent un rôle primordial, car ils interviennent non seulement dans la biologie de chaque espèce mais aussi dans la dynamique de la biocénose toute entière. Cependant, la prolifération et l'abondance des stades larvaires dans les gîtes dépendent des facteurs physico-chimiques tels que la température, la composition chimique de l'eau du gîte, les mouvements de l'eau et la pollution.

Afin d'optimiser le contrôle des larves de moustiques dans leur biotope naturel, il a été procédé au cours de la présente étude à une caractérisation physico-chimique et biologique des gites larvaires permanents et semipermanents à Saga.

\section{MATERIEL ET METHODES \\ Zone d'étude}

Cette étude a été réalisée à Saga, un quartier périurbain localisé dans le $4 \mathrm{em}$ Arrondissement communal de la Ville de Niamey, capitale du Niger et qui est, décrite dans la zone hyper-endémique du paludisme. Ces coordonnées géographiques sont : Longitude : $2^{\circ} 06^{\prime} 35^{\prime \prime}$ Est, Latitude: $13^{\circ} 30^{\prime} 49^{\prime \prime}$ Nord. Situé au bord du fleuve Niger, le quartier de Saga est caractérisé par la présence de plusieurs mares permanentes et semipermanentes. La présence du fleuve a permis l'installation d'un aménagement hydro agricole à vocation rizicole et le développement des cultures irriguées. On y dénombre également de nombreux cours d'eau temporaires ou permanents. Il existe également plusieurs canaux de collecte d'eau et une multitude d'autres gîtes créés par les activités humaines (carrières, etc.) qui constituent des gîtes importants de reproduction de moustiques.

\section{Identification et Geo-positionnement des gîtes larvaires}

Notre étude qui s'est déroulée d'octobre 2016 à juillet 2017, s'est basée sur un inventaire des gites temporaires, semipermanents et permanents effectué à Saga par le programme National de Lutte contre le Paludisme (PNLP) dans le cadre de son programme d'activités. Parmi ces gites préalablement recensés à Saga, seuls les gites accessibles et positifs ont été retenus et géo référencés au moyen d'un GPS et représentés à travers logiciel Google Earth version 7.3 (Figure 1).

\section{Les paramètres mesurés Les paramètres physico-chimiques}

Pour chacun des gîtes suivis, il a été procédé à des mesures des paramètres physicochimiques et une évaluation de l'abondance de la faune culicidienne. Ces paramètres ont été déterminés en octobre, décembre, mars et juillet en raison de deux passages par mois. Ainsi à chaque passage, la température, la conductivité, le $\mathrm{pH}$, la teneur en oxygène dissout ont été mesurés et la densité des larves de moustiques est estimée au niveau de chaque gite.

La mesure des paramètres physicochimique a été effectuée à l'aide d'une louche calibrée dotée d'une longue manche et d'un Conductimètre de type Hanna HI 98130 à 4 paramètres (Figure 2). La méthodologie de détermination des caractères physicochimiques utilisée a consisté à effectuer plusieurs séries de prélèvements d'eau dans plusieurs coins du gite avec la louche. Cette eau collectée est transférée dans un bac dans lequel est plongée l'électrode du conductimètre pour 
effectuer les mesures des différents paramètres obtenus après chaque calibrage de l'appareil.

\section{La densité de la faune culicidienne}

La densité des larves de culicidae notamment les Culex et les Anophèles a été déterminée par la méthode du dipping qui consiste à réaliser dix (10) plongées à l'aide d'une louche d'une capacité de $500 \mathrm{ml}$ dans plusieurs endroits du gîte et sans répétition (Figure 3). Après chaque coup de louche, le contenu est transvasé dans un Bac à fond blanc et le dénombrement des larves est estimé pour 10 louchées (Coffinet et al., 2009 ; Talipouo et al., 2017).

Les larves de moustiques récoltées au niveau des plusieurs gîtes son ramenées au laboratoire, vont servir à un élevage de masse au laboratoire pour obtenir les stades adultes. La détermination des espèces culicidiennes a été faite à l'aide d'une loupe binoculaire, d'une clé d'identification des femelles d'Anophèles élaborées par Gillies et Coetzee (1987) et enfin une clef d'identification des culicidés en se basant sur un ensemble de critères et de descripteurs microscopiques très précis pour les adultes. Au terme de l'étude, la densité larvaire moyenne des différents gîtes a été estimée.

\section{Traitement des données}

Les données collectées ont été saisies et analysées avec le tableur Excel office professionnel version 2013. Les pourcentages et les effectifs ont été calculés dans le tableur Excel. Le test $t$ de Student au seuil de 5\% a été utilisé sur Excel pour comparer les proportions des larves dans les différents gîtes et entre les saisons.

Le logiciel $\mathrm{R}$ ( $\mathrm{R}$ developpement Core Team, 2018) version 3.5.1 a été utilisé pour une Analyse par Composantes Principales (ACP) afin de déterminer les similitudes et les variations intra et inter des différentes gites. La méthode de Classification Ascendante Hiérarchique (CAH) a été utilisée pour dresser la typologie des gites à travers un graphique.

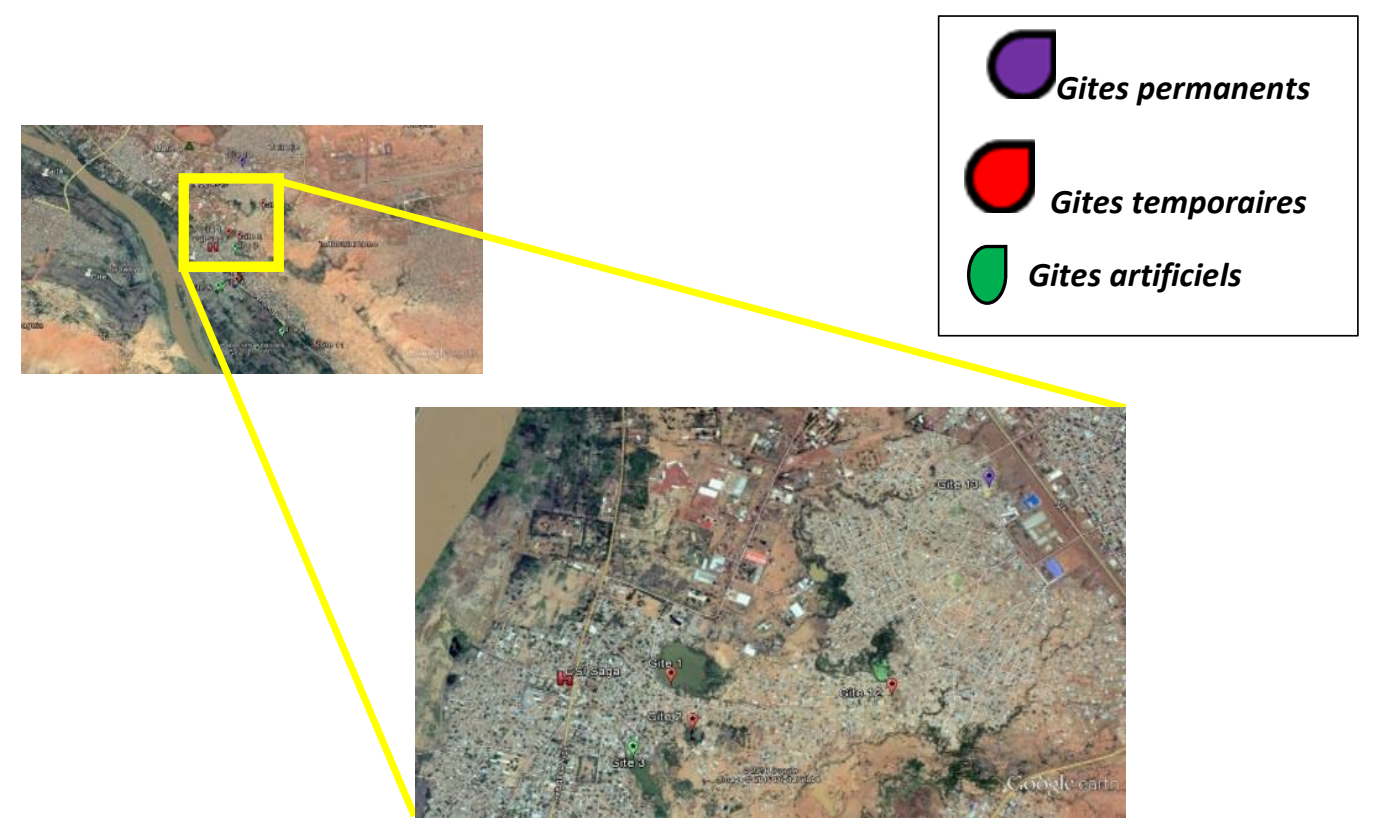

Figure 1: Carte de la région de Niamey montrant la localisation des sites d'étude à Saga. 


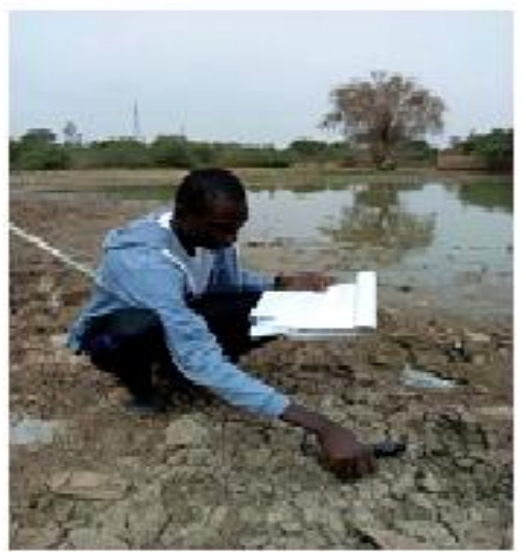

Figure 2: Mesure des paramètres physico-chimiques.

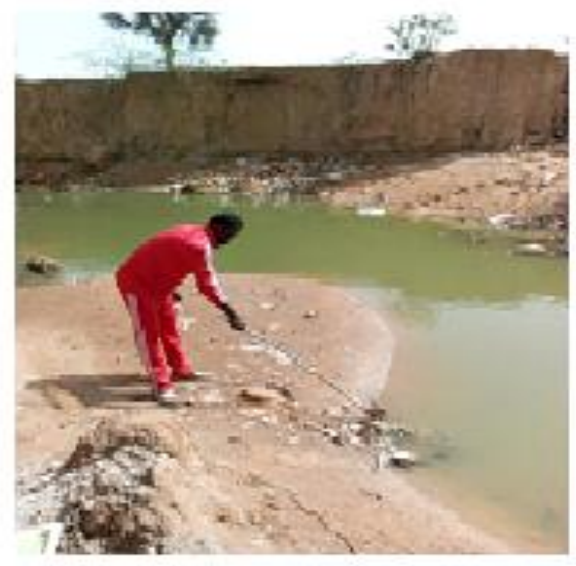

Figure 3 : Mesure de la densité larvaire.

\section{RESULTATS \\ Les gites larvaires}

Au total, 17 gîtes ont été identifiés dans la zone d'étude dont cinq (5) gites temporaires $(29,41 \%)$ et douze (12) gites permanents (Tableau I). Ces gites se répartissent en gites artificiels (6) liés aux activités anthropiques (Briqueterie, carrières...) et en gites naturels tels que les mares, les marigots, les étangs, etc (11).

\section{Peuplement larvaire des gîtes \\ Diversité et densité larvaire moyenne}

L'identification morphologique des larves récoltées au niveau des différentes gîtes a révélé que ces dernières sont composées de larves d'Anopheles gambiae s.l et de Culex $s p$ (Figure 4). Les larves de ces deux espèces ont été observées pendant toutes les périodes de prélèvement. Toutefois, les densités les plus élevées ont été observée en mars et octobre pour ces deux espèces (Figure 4). Cependant, pour une même période, la différence entre la densité larvaire des deux espèces n'est pas significative ( $\mathrm{p}>0,05)$.

\section{Evolution du nombre de gîtes infestés}

Le nombre de gites diminue progressivement de juillet où tous les gîtes sont présents à mars où il ne subsiste que 9 gîtes soit $52,94 \%$ des gîtes identifiés. $94,11 \%$ de ces gîtes sont positifs en octobre, $64,70 \%$ en décembre, $100 \%$ en mars et $52,94 \%$ juillet. (Figure 5). Le pourcentage de gîtes infestés est significativement plus important en octobre et mars qu'en juillet et décembre $(\mathrm{p}<0,05)$.

\section{Caractérisation physicochimique des gites La Température}

La température moyenne de l'eau des gîtes étudiés varie peu au cours de la période d'étude. En effet, ces températures varient de $25,69{ }^{\circ} \mathrm{C}$ au niveau du gîte 9 à $29,37{ }^{\circ} \mathrm{C}$ au niveau du gîte 7 . Toutefois, les températures sont plus faibles en décembre et plus élevées en mars (Tableau 2).

Il n'existe pas de différence significative entre les températures moyennes annuelles enregistrées au niveau des différents gîtes. Il en est de même pour la température moyenne mensuelle des différents gîtes ( $p$ > $0,05)$.

\section{Le pH}

Le $\mathrm{pH}$ mesuré sur l'ensemble des gites est compris entre 6,66 observé au niveau des gîtes 7 et 8,35 observé au niveau du gîte 10 (Tableau 3). Un pH légèrement acide a été observé dans les gites 6 et 14. Mais la plupart des gites étudiés ont un $\mathrm{pH}$ légèrement alcalin.

\section{La conductivité}

La conductivité moyenne oscille entre 0,26 au niveau gîte 14 et $1,44 \mathrm{mS} / \mathrm{cm}$ au niveau du gîte 11. En général, les valeurs les plus élevées ont été enregistrées durant le mois de décembre (valeur moyenne $1,615 \mathrm{mS} / \mathrm{cm}$ ) et les plus faibles sont enregistrées au mois de juillet (Tableau 4). Il existe une différence significative entre la conductivité des gites observée au cours des différentes périodes de mesure $(\mathrm{P}<0.05)$.

Tableau 4: Valeurs de la conductivité moyenne relevées dans les gîtes au cours de la période d'étude. 


\section{Le taux d'oxygène dissout}

La teneur en oxygène mesurée dans l'ensemble des gites est comprise entre 0,05 et 2,32 mg/L. Les valeurs les plus élevées du taux d'oxygène dissout ont été observée durant le mois de mars $(P=0,0042)$. D'une manière générale, le taux d'oxygène dissout a été relativement faible au niveau de tous les gites suivis et durant toute la période d'étude (Tableau 5). Il existe une différence significative entre les taux d'oxygéné dissout enregistré en octobre, décembre, mars et juillet $(\mathrm{P}<0.05)$. Cependant, il n'existe pas de différence significative entre les gîtes pour une même période.

\section{Corrélation entre paramètres physico- chimiques et type de larves}

L'analyse en composante principales (ACP) (Figure 6) présente des corrélations entre les paramètres physicochimiques et la densité larvaire de An. gambiae s.l et culex pipens. Ces données sont représentées sur deux dimensions (Tableau 6). Il ressort de ces données, que les larves d'Anophèles sont fortement corrélées avec le taux d'oxygène dissout. En effet, ces deux paramètres sont tous les deux corrélés avec l'axe 2 (coefficient $\mathrm{R}$ respectivement égale 0,92 et 0,805$)$. Par contre, il exister une relation entre les larves de Culex, la conductivité et le $\mathrm{pH}$. En effet, ces trois paramètres sont fortement corrélés avec l'axe 1 (coefficient $\mathrm{R}$ respectivement $0,818,0,85$ et $0,90)$.

\section{Typologie des gites étudiés}

Les résultats obtenus par la méthode de Classification Ascendante Hiérarchique (CAH) a permis de classer les gites et de les grouper à travers un dendrogramme (Figure 7) obtenu à partir de l'Analyse en Composante Principale (ACP). Les résultats obtenus nous ont permis de grouper les gîtes en 3 types (Tableau 7). gîtes :

Ainsi on distingue trois catégories de

Le groupe 1 constitué par des gîtes : 2 , 6,12 et 14 (coefficient $\mathrm{R}$ respective $0,73,0,43$ $0,62$ et 0,74$)$. Ces gîtes présentent une température comprise entre $29,1^{\circ} \mathrm{C}$ et $30,93{ }^{\circ} \mathrm{C}$ et un $\mathrm{pH}$ légèrement acide à neutre $(\mathrm{pH}$ moins élevé est 6,075 et le plus élevé est 7,035) et ne sont fréquentés que par des larves d'anophèles. Ces gîtes sont constitués pour la plupart des carrieres ou briquetteries.

Le groupe 2 qui est constitué par des gîtes: 1, 3, 4, 5, 9, 11, 13, 16 et 17 (coefficient $\mathrm{R}$ compris entre 0,62 et 0,93 ). Ces gîtes présentent une conductivité plus élevée (comprise entre 1530 et $2250 \mu \mathrm{S} / \mathrm{cm}$ ) ainsi qu'un $\mathrm{pH}$ élevé. Ces gîtes ne sont fréquentés que par des larves de Culex sp en abondance.

Le groupe 3 : qui est constitué par des gîtes 7,10 , et 15 . Dans ces gîtes, on note la présence des trois paramètres élevés : Le $\mathrm{pH}$ (mini 8,02et maxi 8,36), le taux d'oxygène dissout (mini 1,43 mg/l et maxi 2,54 mg/l) et de la conductivité $(1170 \mu \mathrm{S} / \mathrm{cm}$ et $1370 \mu \mathrm{S} / \mathrm{cm})$. Ces gîtes sont caractérisés par la présence des larves de Culex (mini 320 et maxi 732) et d'Anophèles (DL Anophèles mini 285 et maxi 725) en abondance.

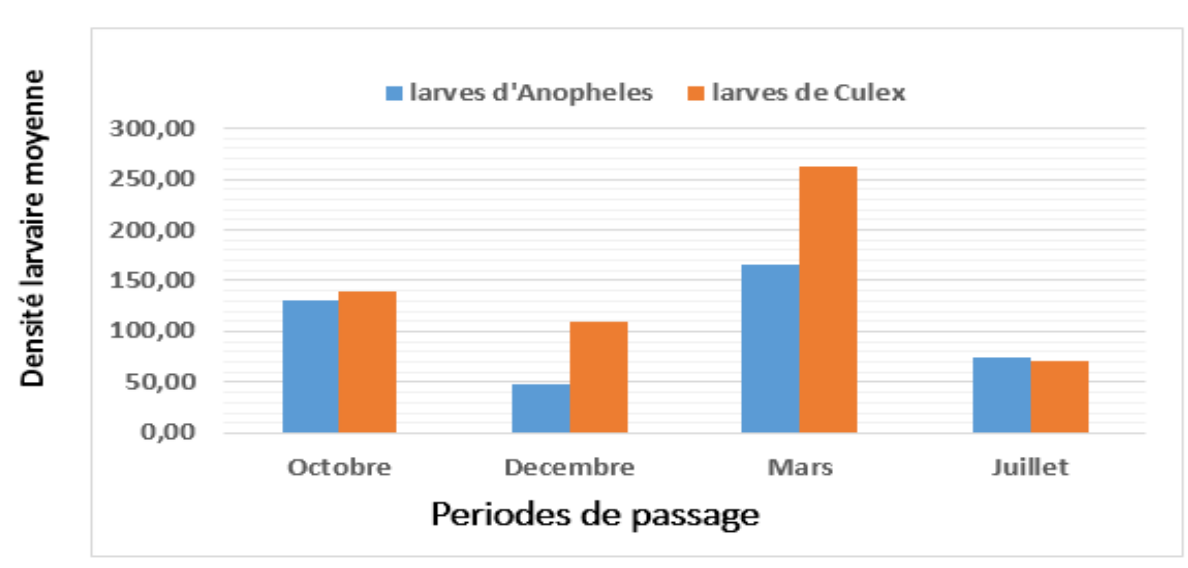

Figure 4: Variation saisonnière de la densité larvaire de An. gambiae s.l en rouge et culex pipens en bleue collectées dans les différents gites de Saga. 
Tableau 1 : Type de gites observés et Coordonnées géographiques à Saga.

\begin{tabular}{|c|c|c|c|c|}
\hline \multirow{2}{*}{$\mathrm{N}^{0}$ du gite } & \multirow{2}{*}{$\begin{array}{l}\text { Caractères } \\
\text { du gite }\end{array}$} & \multirow{2}{*}{$\begin{array}{l}\text { Types de } \\
\text { gite }\end{array}$} & \multicolumn{2}{|c|}{ Coordonnées GPS } \\
\hline & & & Nord & Est \\
\hline Gite 1 & Permanent & Naturel & $13^{\circ} 28^{\prime} 34.27392^{\prime \prime} \mathrm{N}$ & $2^{\circ} 8^{\prime} 37.12848 " \mathrm{E}$ \\
\hline Gite 2 & Permanent & Artificiel & $13^{\circ} 28^{\prime} 21.25704^{\prime \prime} \mathrm{N}$ & $2^{\circ} 8^{\prime} 42.62496 " \mathrm{E}$ \\
\hline Gite 3 & Permanent & Naturel & $13^{\circ} 28^{\prime} 43.92444^{\prime \prime} \mathrm{N}$ & $2^{\circ} 9^{\prime} 0.30852^{\prime \prime} \mathrm{E}$ \\
\hline Gite 4 & Temporaire & Naturel & $13^{\circ} 28^{\prime} 46.3296^{\prime \prime} \mathrm{N}$ & $2^{\circ} 8^{\prime} 55.89708^{\prime \prime} \mathrm{E}$ \\
\hline Gite 5 & Temporaire & Naturel & $13^{\circ} 29^{\prime} 4.23204^{\prime \prime} \mathrm{N}$ & $2^{\circ} 8^{\prime} 57.469592 " \mathrm{E}$ \\
\hline Gite 6 & Temporaire & Artificiel & $13^{\circ} 27^{\prime} 48.77676^{\prime \prime} \mathrm{N}$ & $2^{\circ} 8^{\prime} 34.07712^{\prime \prime} \mathrm{E}$ \\
\hline Gite 7 & Permanent & Artificiel & $13^{\circ} 26^{\prime} 53.76804^{\prime \prime} \mathrm{N}$ & $2^{\circ} 9^{\prime} 20.92176 " \mathrm{E}$ \\
\hline Gite 8 & Temporaire & Naturel & $13^{\circ} 26^{\prime} 44.09772^{\prime \prime N}$ & $2^{\circ} 9^{\prime} 32.62968^{\prime \prime} \mathrm{E}$ \\
\hline Gite 9 & Permanent & Naturel & $13^{\circ} 26^{\prime} 10.3776 " \mathrm{~N}$ & $2^{\circ} 9^{\prime} 53.88228^{\prime \prime} \mathrm{E}$ \\
\hline Gite 10 & Permanent & Artificiel & $13^{\circ} 26^{\prime} 28.36068^{\prime \prime} \mathrm{N}$ & $2^{\circ} 9^{\prime} 14.81904 " \mathrm{E}$ \\
\hline Gite 11 & Temporaire & Naturel & $13^{\circ} 29^{\prime} 17.87208^{\prime \prime} \mathrm{N}$ & $2^{\circ} 8^{\prime} 51.61272^{\prime \prime E}$ \\
\hline Gite 12 & Permanent & Artificiel & $13^{\circ} 28^{\prime} 10.65036^{\prime \prime N}$ & $2^{\circ} 6 ’ 29.92032 " \mathrm{E}$ \\
\hline Gite 13 & Permanent & Naturel & $13^{\circ} 29^{\prime} 49.58484^{\prime \prime} \mathrm{N}$ & $2^{\circ} 5^{\prime} 56.9736 " \mathrm{E}$ \\
\hline Gite 14 & Permanent & Naturel & $13^{\circ} 34^{\prime} 3.20016^{\prime \prime} \mathrm{N}$ & $2^{\circ} 6^{\prime} 32.77728^{\prime \prime} \mathrm{E}$ \\
\hline Gite 15 & Permanent & Artificiel & $\mathrm{N}: 1347878$ & E: 00214912 \\
\hline Gite 16 & Permanent & Naturel & $\mathrm{N}: 1350853$ & E: 00221109 \\
\hline Gite 17 & Permanent & Naturel & $\mathrm{N}: 1354266$ & E: 00222813 \\
\hline
\end{tabular}

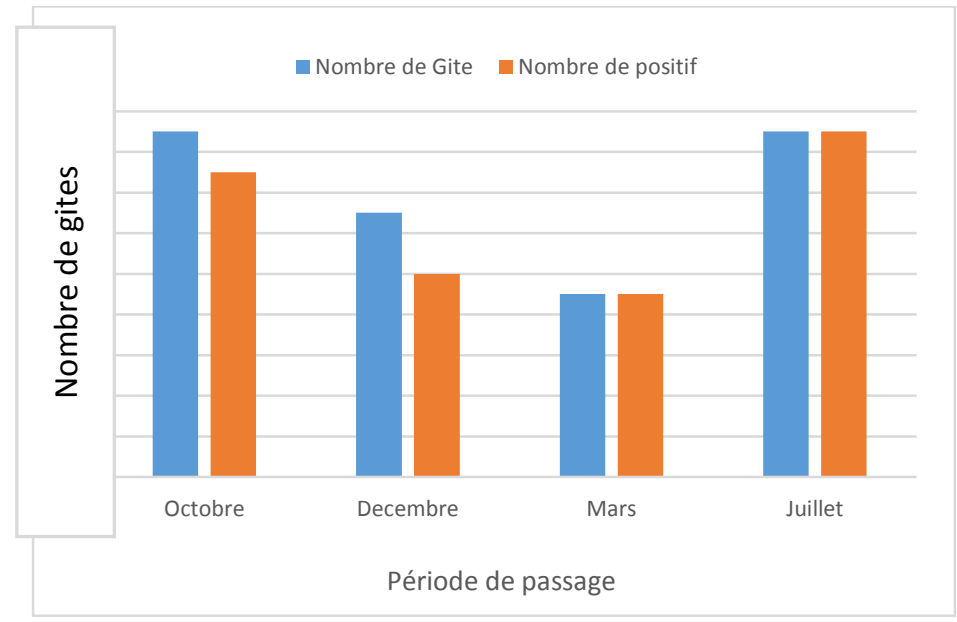

Figure 5 : Variation saisonnier et infestation des gites. 
Tableau 2: Valeurs des températures relevées dans les gîtes au cours de la période d'étude.

\begin{tabular}{|c|c|c|c|c|c|}
\hline Gites & Octobre & Décembre & Mars & Juillet & Moyenne \\
\hline Gite 1 & $27,10 \pm 0,71$ & $22,57 \pm 0,93$ & $30,27 \pm 1,44$ & $30,17 \pm 1,70$ & 27,53 \\
\hline Gite 2 & $27,25 \pm 1,48$ & $23,7 \pm 0,56$ & $29,43 \pm 0,25$ & $29,6 \pm 0,92$ & 27,50 \\
\hline Gite 3 & $27,00 \pm 0,71$ & $19,83 \pm 0,93$ & $30,10 \pm 0,56$ & $31,4 \pm 1,85$ & 27,08 \\
\hline Gite 4 & $27,45 \pm 1,77$ & $22,87 \pm 1,88$ & - & $28,4 \pm 2,1$ & 26,24 \\
\hline Gite 5 & $29,50 \pm 1,13$ & $23 \pm 0,7$ & - & $31,2 \pm 1,77$ & 27,90 \\
\hline Gite 6 & $28,80 \pm 0,85$ & $21,60 \pm 1,37$ & - & $28,17 \pm 1,93$ & 26,19 \\
\hline Gite 7 & $31,75 \pm 0,78$ & $22,90 \pm 4,48$ & $33,27 \pm 0,21$ & $29,57 \pm 1,66$ & 29,37 \\
\hline Gite 8 & $30,35 \pm 0,92$ & $23,33 \pm 6$ & - & $30,13 \pm 1,44$ & 27,94 \\
\hline Gite 9 & $25,45 \pm 0,92$ & $20 \pm 3,22$ & $29,6 \pm 1,76$ & $27,7 \pm 2,01$ & 25,69 \\
\hline Gite 10 & $29,40 \pm 0,85$ & $21,47 \pm 4,14$ & $30,87 \pm 1,46$ & $29,2 \pm 1,23$ & 27,74 \\
\hline Gite 11 & $29,30 \pm 4,53$ & $22,20 \pm 4,33$ & $29,87 \pm 0,83$ & $29,73 \pm 1,50$ & 27,78 \\
\hline Gite 12 & $27,45 \pm 1,91$ & $19,73 \pm 1,65$ & - & $31,67 \pm 3,23$ & 26,28 \\
\hline Gite 13 & $27,90 \pm 0,71$ & $21,27 \pm 4,77$ & $30,10 \pm 1,37$ & $27,12 \pm 1,31$ & 26,60 \\
\hline Gite 14 & $26,80 \pm 1,13$ & $23,47 \pm 4,88$ & $30,37 \pm 1,50$ & $28,57 \pm 1,11$ & 27,30 \\
\hline Gite 15 & $28,90 \pm 0,42$ & $22,43 \pm 2,94$ & $28,73 \pm 1,27$ & $28,7 \pm 0,9$ & 27,19 \\
\hline Gite 16 & $27,90 \pm 0,42$ & $23,43 \pm 0,57$ & $28,73 \pm 0,91$ & $28,6 \pm 1,8$ & 27,17 \\
\hline Gite 17 & $29,35 \pm 1,2$ & $22,80 \pm 0,95$ & $31,67 \pm 2,16$ & $31,33 \pm 1,36$ & 28,79 \\
\hline $\begin{array}{l}\text { Statistique } \\
\text { de Fisher }\end{array}$ & \multicolumn{5}{|c|}{1.775} \\
\hline P-Value & \multicolumn{5}{|c|}{0.173 (non significative) } \\
\hline Légende & \multicolumn{5}{|c|}{$(-)=$ absence de données pour tarissement des gites durant la période } \\
\hline
\end{tabular}

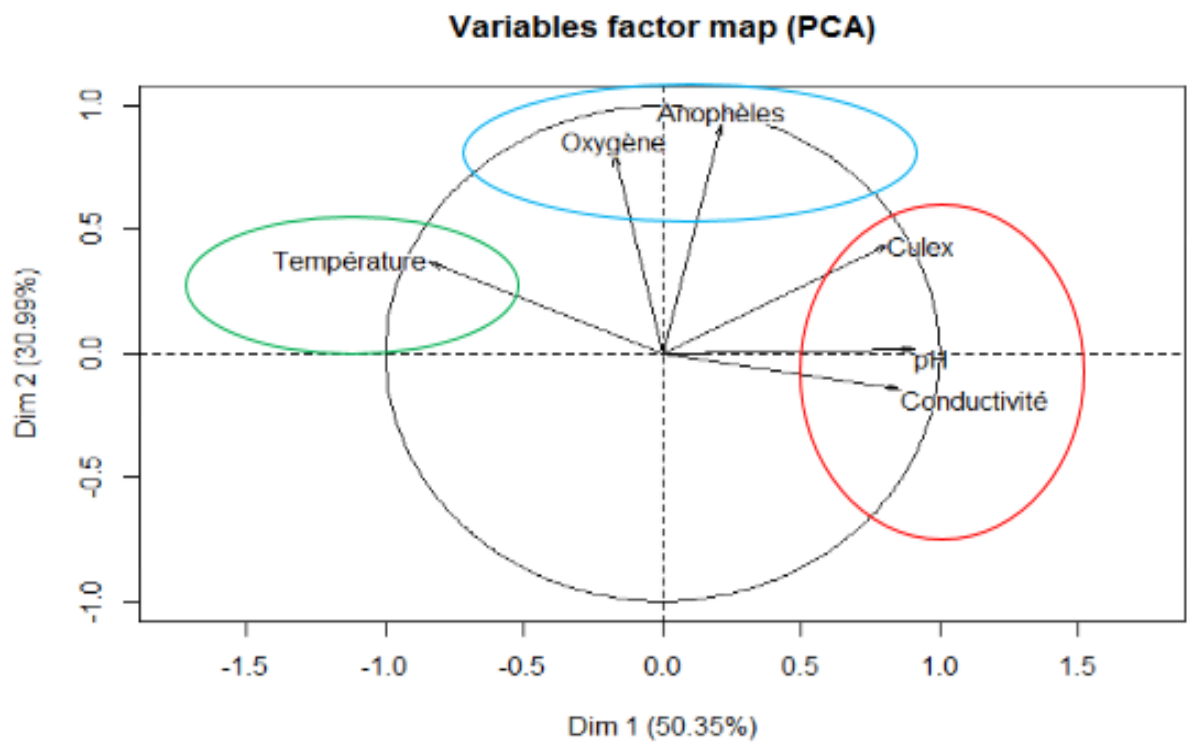

Figure 6: Représentation graphique de la projection des paramètres physico-chimique des variables (la densité larvaire des Anophèles et des Culex) dans le même plan F1 x F2 de l'A.C.P 
Tableau 3: Valeurs du pH relevées dans les gîtes au cours de la période d'étude.

\begin{tabular}{|c|c|c|c|c|c|}
\hline Gites & Octobre & Décembre & Mars & Juillet & Moyenne annuelle \\
\hline G1 & $7,02 \pm 0,28$ & $7,91 \pm 0,05$ & $7,45 \pm 0,23$ & $7,68 \pm 0,39$ & 7,51 \\
\hline G2 & $6,78 \pm 0,28$ & $6,75 \pm 0,32$ & $7,44 \pm 0,22$ & $7,39 \pm 0,18$ & 7,09 \\
\hline G3 & $8,21 \pm 0,59$ & $8,38 \pm 0,37$ & $7,48 \pm 0,23$ & $7,27 \pm 0,14$ & 7,835 \\
\hline G4 & $7,54 \pm 0,5$ & $8,16 \pm 0,43$ & - & $7,8 \pm 0,75$ & 7,83 \\
\hline G5 & $7,56 \pm 0,54$ & $7,88 \pm 0,02$ & - & $7,92 \pm 0,68$ & 7,78 \\
\hline G6 & $6,62 \pm 0,18$ & $6,57 \pm 0,50$ & - & $6,79 \pm 0,04$ & 6,66 \\
\hline G7 & $7,65 \pm 0,23$ & $8,22 \pm 0,41$ & $8,11 \pm 0,14$ & $8,09 \pm 0,31$ & 8,0175 \\
\hline G8 & $7,57 \pm 0,18$ & $8,43 \pm 0,56$ & - & $7,32 \pm 0,33$ & 7,77 \\
\hline G9 & $7,36 \pm 0,88$ & $7,97 \pm 0,03$ & $7,97 \pm 1,36$ & $7,18 \pm 0,39$ & 7,62 \\
\hline G10 & $8,39 \pm 0,3$ & $8,18 \pm 0,63$ & $8,66 \pm 0,08$ & $8,2 \pm 0,08$ & 8,3575 \\
\hline G11 & $7,51 \pm 0,34$ & $8,62 \pm 0,3$ & - & $7,23 \pm 0,12$ & 7,78 \\
\hline G12 & $7,76 \pm 0,01$ & $7,3 \pm 0,49$ & $6,88 \pm 0,13$ & $7,07 \pm 0,19$ & 7,25 \\
\hline G13 & $6,91 \pm 0,33$ & $7,88 \pm 0,07$ & $7,52 \pm 0,14$ & $7,25 \pm 0,36$ & 7,39 \\
\hline G14 & $7,51 \pm 0,28$ & $6,15 \pm 0,18$ & $7,04 \pm 0,22$ & $7,02 \pm 0,26$ & 6,93 \\
\hline$G 15$ & $7,84 \pm 0,34$ & $8,35 \pm 0,14$ & $8,28 \pm 0,25$ & $8,47 \pm 0,21$ & 8,23 \\
\hline G16 & $7,26 \pm 0,08$ & $6,87 \pm 0,26$ & $6,93 \pm 0,08$ & $7,33 \pm 0,08$ & 7,09 \\
\hline G17 & $7,18 \pm 0,08$ & $7,22 \pm 0,05$ & $6,95 \pm 0,14$ & $7,36 \pm 0,26$ & 7,17 \\
\hline Moyenne & 7,37 & 7,71 & 7,24 & 7,31 & 7,40 \\
\hline $\begin{array}{l}\text { Statistique } \\
\text { de Fisher }\end{array}$ & \multicolumn{5}{|c|}{0.0355} \\
\hline p-value & \multirow{2}{*}{\multicolumn{5}{|c|}{0.9651 (Non significative) }} \\
\hline Légende & & & & & \\
\hline
\end{tabular}

Tableau 4 : Valeurs de la conductivité moyenne relevées dans les gîtes au cours de la période d'étude.

\begin{tabular}{|c|c|c|c|c|c|}
\hline Gites & Octobre & Décembre & Mars & Juillet & Moyenne annuelle \\
\hline Gite 1 & $0,82 \pm 0,28$ & $1,88 \pm 0,08$ & $0,975 \pm 0,47$ & $0,52 \pm 0,01$ & 1,05 \\
\hline Gite 2 & $0,29 \pm 0,2$ & $0,59 \pm 0,14$ & $1,27 \pm 1,023$ & $0,15 \pm 0,006$ & 0,58 \\
\hline Gite 3 & $1,32 \pm 0,03$ & $1,31 \pm 0,13$ & $0,89 \pm 0,43$ & $0,77 \pm 0,03$ & 1,07 \\
\hline Gite 4 & $0,99 \pm 0,19$ & $1,35 \pm 0,34$ & - & $0,69 \pm 0,02$ & 1,01 \\
\hline Gite 5 & $1,29 \pm 0,23$ & $1,44 \pm 0,52$ & - & $0,52 \pm 0,06$ & 1,08 \\
\hline Gite 6 & $0,34 \pm 0,06$ & $0,94 \pm 0,75$ & - & $0,32 \pm 0,13$ & 0,53 \\
\hline Gite 7 & $1,17 \pm 0,12$ & $1,73 \pm 0,08$ & $1,37 \pm 0,43$ & $1,22 \pm 0,1$ & 1,37 \\
\hline Gite 8 & $0,17 \pm 0,04$ & $1,4 \pm 0,35$ & - & $0,09 \pm 0,02$ & 0,55 \\
\hline Gite 9 & $0,98 \pm 0,29$ & $1,61 \pm 0,27$ & $0,83 \pm 0,843$ & $0,6 \pm 0,04$ & 1,01 \\
\hline Gite 10 & $1,19 \pm 0,07$ & $2,24 \pm 0,05$ & $0,81 \pm 0,13$ & $1,514 \pm 0,02$ & 1,44 \\
\hline Gite 11 & $1,17 \pm 0,39$ & $1,52 \pm 0,25$ & - & $0,47 \pm 0,06$ & 1,05 \\
\hline Gite 12 & $0,58 \pm 0,23$ & $1,6 \pm 1,09$ & $0,24 \pm 0,26$ & $0,510,22$ & 0,73 \\
\hline Gite 13 & $0,74 \pm 0,13$ & $1,44 \pm 0,64$ & $1,175 \pm 0,87$ & $0,52 \pm 0,15$ & 0,97 \\
\hline Gite 14 & $0,19 \pm 0,11$ & $0,42 \pm 0,31$ & $0,25 \pm 0,41$ & $0,16 \pm 0,01$ & 0,26 \\
\hline
\end{tabular}


S. MAHAMANE IRO et al. / Int. J. Biol. Chem. Sci. 14(4): 1188-1202, 2020

\begin{tabular}{r|ccccr} 
Gite 15 & $1,14 \pm 0,28$ & $1,8 \pm 0,11$ & $0,9 \pm 0,26$ & $0,85 \pm 0,05$ & 1,17 \\
Gite 16 & $0,95 \pm 0,01$ & $0,89 \pm 0,07$ & $1,09 \pm 0,53$ & $0,4 \pm 0,1$ & 0,83 \\
Gite 17 & $0,76 \pm 0,01$ & $1,31 \pm 0,09$ & $0,8 \pm 0,04$ & $0,7 \pm 0,02$ & 0,89 \\
Moyenne & 0,83 & 1,38 & 0,71 & 0,24 & $\mathbf{0 , 7 9}$ \\
Statistique de & 4.214 & \\
Fisher & \multicolumn{5}{c}{$0.0164<0.05$ (Significative) * } \\
P-Value & $(-)=$ absence de données pour tarissement des gites durant la période \\
Légende & \multicolumn{4}{c}{}
\end{tabular}

Tableau 5: Valeurs du taux d'oxygène dissout relevées dans les gîtes dans nos conditions expérimentales.

\begin{tabular}{|c|c|c|c|c|c|}
\hline Les gites & Octobre & Décembre & Mars & Juillet & Moyenne annuelle \\
\hline Gite1 & $1,04 \pm 0,28$ & $0,83 \pm 0,28$ & $0,94 \pm 0,35$ & $0,44 \pm 0,03$ & 0,813 \\
\hline Gite 2 & $1,02 \pm 0,18$ & $1,29 \pm 0,93$ & $2,32 \pm 0,46$ & $0,29 \pm 0,01$ & 1,230 \\
\hline Git3 & $0,45 \pm 0,06$ & $0,45 \pm 0,03$ & $0,86 \pm 0,48$ & $0,34 \pm 0,01$ & 0,525 \\
\hline Gite 4 & $0,84 \pm 0,37$ & $1,32 \pm 0,44$ & - & $0,58 \pm 0,04$ & 0,913 \\
\hline Gite 5 & $1,17 \pm 0,84$ & $1,54 \pm 0,84$ & - & $0,44 \pm 0,11$ & 0,717 \\
\hline Gite 6 & $1,01 \pm 0,47$ & $1,36 \pm 0,61$ & - & $0,64 \pm 0,25$ & 1,003 \\
\hline Gite 7 & $0,8 \pm 0,23$ & $1,74 \pm 0,2$ & $1,09 \pm 0,68$ & $0,74 \pm 0,19$ & 1,093 \\
\hline Gite 8 & $0,4 \pm 0,08$ & $1,75 \pm 0,97$ & - & $0,18 \pm 0,04$ & 0,777 \\
\hline Gite 9 & $0,44 \pm 1,24$ & $0,88 \pm 0,69$ & $1,69 \pm 1,19$ & $0,4 \pm 0,07$ & 0,853 \\
\hline Gite 10 & $1,28 \pm 0,15$ & $0,93 \pm 0,15$ & $2,54 \pm 0,78$ & $0,85 \pm 0,025$ & 1,400 \\
\hline Gite 11 & $0,9 \pm 0,77$ & $0,6 \pm 0,43$ & - & $0,23 \pm 0,04$ & 0,577 \\
\hline Gite 12 & $1,83 \pm 0,46$ & $1,81 \pm 0,47$ & $2,49 \pm 0,01$ & $0,85 \pm 0,03$ & 1,745 \\
\hline Gite 13 & $0,3 \pm 0,43$ & $1,15 \pm 0,92$ & $1,07 \pm 0,05$ & $0,49 \pm 0,17$ & 0,753 \\
\hline Gite 14 & $0,81 \pm 0,43$ & $0,83 \pm 0,63$ & $1,96 \pm 0,09$ & $0,43 \pm 0,2$ & 1,008 \\
\hline Gite 15 & $0,87 \pm 0,11$ & $0,96 \pm 0,31$ & $2,43 \pm 0,26$ & $0,76 \pm 0,02$ & 1,255 \\
\hline Gite 16 & $0,76 \pm 0,06$ & $0,96 \pm 0,86$ & $1,42 \pm 0,56$ & $0,13 \pm 0,06$ & 0,818 \\
\hline Gite 17 & $0,62 \pm 0,35$ & $0,69 \pm 0,27$ & $0,05 \pm 0,02$ & $0,08 \pm 0,11$ & 0,360 \\
\hline Moyenne & 0,796470588 & 1,122941176 & 1,257333333 & 0,46294118 & 0,910 \\
\hline $\begin{array}{r}\text { Statistique } \\
\text { de Fisher } \\
\text { P-Value }\end{array}$ & \multicolumn{5}{|c|}{$\begin{array}{c}2.526 \\
0.083 \text { (Non significative) }\end{array}$} \\
\hline Légende & \multicolumn{5}{|c|}{$(-)=$ absence de données pour tarissement des gites durant la période } \\
\hline
\end{tabular}


Tableau 6: Corrélations entre les variables et les gites.

\begin{tabular}{lcc}
\hline & Dimenssion.1 & Dimenssion.2 \\
\hline Anophèles sp & 0,2130236 & 0,92721693 \\
Culex sp & 0,8187085 & 0,44077806 \\
Température & $-0,8435495$ & 0,36978726 \\
pH & 0,9085225 & 0,01748034 \\
Oxygène dissout & $-0,1745391$ & 0,8051219 \\
Conductivité & $-0,8589768$ & $-0,14180821$ \\
\hline
\end{tabular}

Tableau 7 : Corrélation des gites et densité larvaire.

\begin{tabular}{lll}
\hline Gites corrélés avec les larves d'Anophèles & Gites corrélés avec les larves de Culex & Gites intermédiaires \\
\hline Gites $2(\mathbf{R}=\mathbf{0 , 7 3 5 2})$ & Gite $1(\mathrm{R}=0,7555)$ & Gite $7(\mathrm{R}=0,4$ et $\mathrm{O}, 22)$ \\
\hline Gites $\mathbf{6}(\mathbf{R}=\mathbf{0 , 6 2 0 6 5})$ & Gite $3(\mathrm{R}=0,6242)$ & Gite $10(\mathrm{R}=0,21$ et 0,25$)$ \\
\hline Gites $12(\mathbf{R}=\mathbf{0 , 7 3 1 6 5 5})$ & Gite $4(\mathrm{R}=0,6876)$ & Gite $15(\mathrm{R}=0,50$ et 0,37$)$ \\
\hline Gites $\mathbf{1 4}(\mathbf{R}=\mathbf{0 , 7 4 6 2 0 3})$ & Gite $5(\mathrm{R}=0,642086)$ & \\
\hline & Gite $9(\mathrm{R}=0,856726)$ & \\
\hline & Gite $11(\mathrm{R}=0,864083)$ & \\
\hline & Gite $13(\mathrm{R}=0,867883)$ & \\
\hline & Gite $16(\mathrm{R}=0,745567)$ & \\
\hline & Gite $17(\mathrm{R}=0,936499)$ & \\
\hline
\end{tabular}

\section{Cluster Dendrogram}

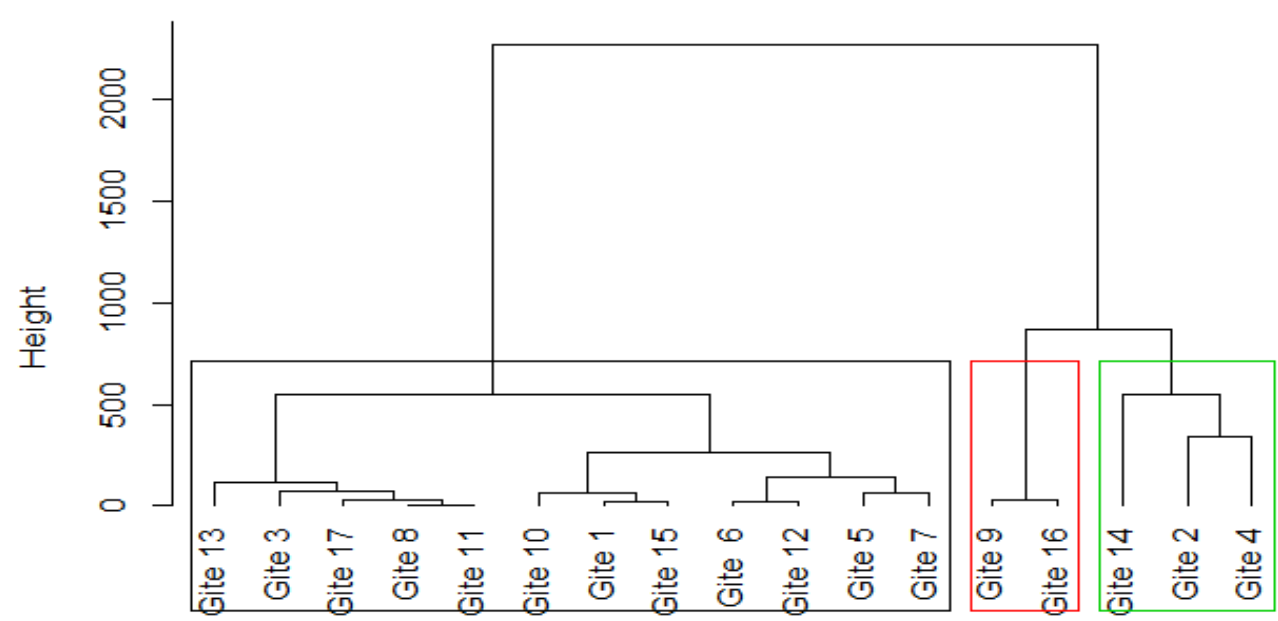

dist(patenew)

hclust (", "ward.D")

Figure 7 : Dendrogramme analyse de groupements des gites. 


\section{DISCUSSION}

Les travaux réalisés au cours de cette étude ont permis de recenser 17 gîtes larvaires au niveau du Quartier de Saga dont 11 gîtes naturels et 6 gîtes artificiels. Il ressort ainsi que le nombre de gîtes artificiels en lien avec les activités anthropiques (briqueterie, cultures irriguées, etc.) sont relativement faibles contrairement à une étude similaire réalisée à Mouila (Sud-Ouest du Gabon) par Koumba et al. (2018) qui ont dénombré 122 gîtes artificiels sur 178 recensés. Mais la particularité de ces gîtes artificiels est d'héberger en abondance des larves de culex et d'anophèles contrairement aux gîtes naturels. Nos résultats sont similaires à ceux de Tia et al. (2016) qui ont montré que les populations, en contribuant à la prolifération des gîtes larvaires, sont responsables de la mise en place des conditions propices au développement et au maintien des moustiques. Le suivi de ces gites a montré que le nombre total de gîtes larvaires varie en fonction des saisons. Ce nombre est plus élevé en saison pluvieuse (juillet et octobre) qu'en saison sèche (mars). Ceci s'explique par les conditions drastiques du climat sahélien qui entraine un tarissement de la plupart des mares temporaires quelques mois après la fin des pluies. Par contre, le nombre de gîtes positifs est plus élevé pendant la saison sèche qu'en saison des pluies. Des résultats similaires ont été obtenus par Gerard et al. (2007), Sy et al. (2016) et Koumba et al. (2018) dans des régions similaires (péri-urbaines) qui ont observé de faibles proportions de gîtes positifs en saison des pluies contrairement en saison sèche. Cette faible proportion de gîtes positifs observée en saison des pluies, pourrait s'expliquer par les perturbations et le lessivage constants de ces gîtes par les pluies. Ces résultats pourraient aussi s'expliquer par la rareté des gîtes larvaires pendant la saison sèche donnant ainsi peu de choix aux femelles qui pondraient leurs œufs dans le peu de gîtes disponibles.

Par ailleurs, seules les larves d'Anophèles gambiae s.l et de Culex pipens ont été collectées au cours de cette étude. La densité de ces larves est plus faible en saison des pluies (moins de 100 larves/10 louchées) qu'en saison sèche (plus de 500 larves/10 louchées). Ces résultats sont contraires à ceux de Gérard et al. (2007), de Koumba et al. (2018) respectivement au Gabon et ainsi que l'étude de Sy et al. (2016) au Sud du Sénégal, qui ont montré que la production larvaire culicidienne est plus élevée en saison pluvieuse qu'en saison sèche. Selon ces auteurs, cette infestation des gîtes pourrait s'expliquer, d'une part, par la forte quantité des gîtes larvaires permanents présents dans la zone d'étude et, d'autre part, au fait que la saison pluvieuse offre des possibilités de choix des gîtes aux femelles des différentes espèces de moustiques aussi bien en qualité qu'en quantité. Or, cela n'est pas le cas pour la saison sèche où certains gîtes favorables au développement des moustiques s'assèchent ou disparaissent.

La caractérisation des paramètres physico-chimiques des gîtes larvaires ne montre aucune différence significative entre les températures moyennes annuelles enregistrées au niveau des différents gîtes d'une part, et d'autre part, entre les températures moyennes mensuelles des différents gîtes. Les températures enregistrées au cours de cette étude (comprise entre $19,73{ }^{\circ} \mathrm{C}$ et $33,27{ }^{\circ} \mathrm{C}$ ) sont des valeurs favorables au développement de plusieurs espèces de moustiques tels que mentionnés par plusieurs auteurs (Messai et al. 2011, El Ouali Lalami et al. 2010, Ikram et al. (2016). Nos résultats ont révélé que les températures maximales ont été observées durant le mois de mars $\left(33,27^{\circ} \mathrm{C}\right.$ dans le gîtes 7) et la densité larvaire des Anophèles demeurent la plus élevée durant cette même période (166 larves/lire en moyenne en mars contre 74,04 en Juillet, 131,38 en Octobre et 48,71 en décembre). Ces résultats sont similaires à ceux obtenus par Martine et al., (2014), qui ont pu démontrer également que le développement larvaire de An. gambiae s.l est plus rapide à une température moyenne de 32 ${ }^{\circ} \mathrm{C}$.

Par contre, la conductivité moyenne et le taux moyen d'oxygène dissout diffèrent significativement en fonction des différentes périodes de mesure. Cependant, aucune différence significative n'est observée entre les gîtes pour une même période au niveau de ces 
gîtes. Pour l'ensemble des gites étudiés, la conductivité est relativement faible (valeurs comprises entre 0,06 et $1,615 \mathrm{mS} /$ ) contrairement aux études de Ikram et al. (2016) qui ont obtenu des valeurs plus élevées (comprises entre 100 et $9400 \mu \mathrm{S} / \mathrm{cm}$ ) au Maroc. Benhissen et al. (2016) ont obtenu des valeurs comprises entre 100 et $1780 \mu \mathrm{S} / \mathrm{cm}$ en Algérie et Tia et al. (2016) avaient obtenu des valeurs tantôt élevées, atteignant $1337 \mu \mathrm{S} / \mathrm{cm}$ ou tantôt faible $(75,87 \mu \mathrm{S} / \mathrm{cm})$.

$\mathrm{La}$ teneur en oxygène dissout de l'ensemble de nos gîtes est en général relativement faible (valeur comprise entre 0,05 et $2,32 \mathrm{mg} / \mathrm{L}$ ). Les études de El Ouali Lalami et al. (2010) et celles de Tia et al. (2016) respectivement en Algérie et en Côte d'ivoire ont obtenu des taux plus élevés $(1,36$ et 9,28 $\mathrm{mg} / \mathrm{L})$.

S'agissant $\mathrm{du} \mathrm{pH}$, nous avons obtenu dans notre étude soit des valeurs de $\mathrm{pH}$ acide avec une tendance neutre $(6,66$ à 7,09$)$, des pH légèrement basiques $(7,78$ à 7,83$)$ et des gîtes à $\mathrm{pH}$ basique $(8,23$ à 8,35$)$. Toutefois, à l'exception des gîtes $2,6,14,16$ et 17 , l'ensemble des gîtes prospectés au cours de cette étude ont un $\mathrm{pH}$ acide. Dans tous ces gîtes prospectés, il a été observé des larves d'anophèles à l'exception des gîtes à $\mathrm{pH}$ basique qui sont colonisés plus abondement que par des larves de culex. Nos études sont similaires à celles de Martine (2014) qui ont recensé les mêmes types de gîtes: soit des gîtes à un $\mathrm{pH}$-moyen, acide $(\mathrm{pH}=6,29)$, neutre $(\mathrm{pH}=7,00)$ et basique $(\mathrm{pH}=7,54)$ mais n'ont pas observé des larves d'Anophèles dans gîtes à $\mathrm{pH}$ basique. Le $\mathrm{pH}$ d'un gîte reflète l'activité biologique du milieu.

Cette étude a montré que les paramètres physico-chimiques ainsi décrits influencent considérablement la répartition et le développement des populations larvaires des culicidae. C'est ainsi que la relation entre ces paramètres et la densité larvaire a permis de distinguer trois types de gîtes :

Les gites du groupe 1 (gîtes 2, 6, 12 et 14) caractérisé par un $\mathrm{pH}$ légèrement acide à neutre ( $\mathrm{pH}$ moins élevé est 6,075 et le plus élevé est 7,035) et un taux d'oxygène dissout élevé (compris entre 1,11 et 2,32). Ces gites ne sont fréquentés que par des larves d'Anophèles. Nos observations concordent avec les études de Ikram et al. (2016) qui ont montré que les larves d'Anophèles préfèrent des eaux bien oxygénées et avec $\mathrm{pH}$ acide.

Les gites du groupe 2 (gîte 1 , gîte 3 , gîte 4 , gîte 5 , gîte 9 , gîte 11 , gîte 13 et gîtes 17 ) sont caractérisés par une conductivité élevée (compris entre 0,97 à 1,08 ) et un $\mathrm{pH}$ légèrement basique $(7,51$ à 7,83). Ces gîtes sont fortement colonisés par des larves de Culex. Des résultats similaires ont et rapportés par Ikram et al. (2016) qui n'ont observé des larves de culex que dans des gites ayant une conductivité comprise entre 600 et 1300 $\mu \mathrm{S} / \mathrm{cm}$. Les résultats obtenus par El Ouali Lalami et al. (2010) au Maroc ont relevé que tous les gîtes à conductivité relativement élevée et peu oxygénés étaient colonisés par des culex.

Les gites du groupe 3 (gîte 7 , gîte 10, et gîte 15): Dans ces gîtes, on note la présence des trois paramètres élevés à savoir le $\mathrm{pH}$ (mini 8,02 et maxi 8,36), le taux d'oxygène dissout (mini $1,43 \mathrm{mg} / \mathrm{l}$ et maxi $2,54 \mathrm{mg} / \mathrm{l}$ ) et de la conductivité $(1170 \mu \mathrm{S} / \mathrm{cm}$ et $1370 \mu \mathrm{S} / \mathrm{cm})$. Ces gites sont caractérisés par la présence des larves de Culex et Anophèles en abondance. Cette étude met l'accent sur la plasticité de ces deux espèces des culicidés observés (An. gambiae et Culex pipens) et leur capacité à pondre et à se développer dans ces types de gîtes.

\section{Conclusion}

En dépit de l'aire limitée de notre prospection entomologique, il apparaît que la distribution des populations des culicidés dans le quartier de Saga, est influencée par les paramètres physicochimiques des gîtes larvaires. C'est ainsi que les anophèles ont montré une grande affinité aux gîtes possédant un taux d'oxygène dissout et une température élevés et un $\mathrm{pH}$ légèrement acide. Alors que les Culex $s p$ présentent une affinité aux gîtes ayant une conductivité élevée et une faible teneur en oxygène. Cette étude biotypologique dans le quartier de saga devrait permettre d'adapter les campagnes de traitement des gîtes larvaires selon les types de gîtes, les espèces et genres 
ciblées et la période propice de pullulation des moustiques.

\section{CONFLIT D'INTERETS}

Les auteurs déclarent ne pas avoir de conflit d'intérêts.

\section{REMERCIEMENTS}

Ce travail a été rendu possible par la disponibilité des Biolarvicides Bactivec et Griselesf fournis par le Programme National de Lutte contre le Paludisme (PNLP) du Niger et de certain matériel fournis par le Centre de Recherche Médical et Sanitaire (CERMES) du Niger.

Nos remerciements à $\mathrm{Mr}$ Adamou Laouali Doctorant en Bio statistique à l'Université Abdou Moumouni de Niamey et Abdoul Aziz Maiga, doctorant en Entomologie médicale à l'université de Ouagadougou qui ont participé au traitement des données (Méthode ACP).

Les auteurs remercient tous ceux qui ont apporté leur aide pour la correction de ce document.

\section{REFERENCES}

Abagli AZ, Alavo TBC, Brodeur J. 2014. Microorganismes entomopathogènes, prédateurs et parasites des moustiques : Perspectives pour la lutte raisonnée contre les vecteurs du paludisme en Afrique sub-saharienne. International Journal of Biological and Chemical Sciences, 8(1): 340-354. DOI: http://dx.doi.org/10.4314/ijbcs.v8i1.29

El Ouali Lalami A, El Hilali O, Benlamlih M, Merzouki M, Raiss N, Ibensouda Koraichi S, Himmi O. 2010. Etude entomologique, physicochimique et bactériologique des gîtes larvaires de localités à risque potentiel pour le paludisme dans la ville de Fès, Maroc. Bulletin de l'Institut Scientifique, Rabat, section Sciences de la Vie, 32 (2) : 119127.

Ahadji-Dabla KM, Ketoh GK, Nyamador WS, Apetogbo GY, Glitho IA. 2014. Susceptibility to DDT and Pyrethroids, and Detection of Knockdown Resistance Mutation in Anopheles Gambiae Sensu Lato in Southern Togo. International Journal of Biological and Chemical
Sciences, $\quad 8(1): \quad 314-23 . \quad$ DOI: 10.4314/ijbcs.v8i1.27.

Benhissen S. 2016. Effet des facteurs écologiques sur l'abondance saisonnière. Essais de lutte. Thèse de Doctorat, Université d'Annaba, Algérie, 135p.

Berchi S, aouati A, Louad K. 2012. Typologie des gîtes propices au développement larvaire de Culex pipiens L. 1758 (Diptera: Culicidae), source de nuisance à Constantine (Algérie). Ecologia Méditeranea, 38 (2) : 5-16.

Brunhes J, Rhaim A, Geoffro B, Angel G, Hervy Jean-Paul. 2000. Les moustiques de l'Afrique méditerranéenne: Espèces présentes et répartition (Diptera, Nematocera). Bulletin de la Société Entomologique de France, 105(2): 195204.

Coffinet T, Rogier C. 2009. Evaluation de l'agressivité des anophèles et du risque de transmission du paludisme : méthodes utilisées dans les armées françaises. Méd Trop, 69 (2) : 109-122.

Gerard G, Caron M, Mombo I, Nkoghe D, Mboui-Ondo S, Jiolle D, Fontenille D, Paupy C, Leroy EM. 2014. Zika Virus in Gabon, Central Africa. A new threat from Aedes albopictus. PLoS Neglected Tropical Diseases, 8(2): 1-6. e2681. DOI: 10.1371/journal.pntd.0002681

Habou ZA, Mahamadou KB, Toudou A. 2016. Les Systèmes de Productions Agricoles $\mathrm{Du}$ Niger Face $\mathrm{Au}$ Changement Climatique : Défis et Perspectives. International Journal of Biological and Chemical Sciences, 10(3): 1262-72. DOI: 10.4314/ijbcs.v10i3.28

Herrel A, Aerts P, De Vree F. 2001. Cranialkinesis in geckoes: Functional implications. J. Exp. Biol., 203: 14151423. DOI: $10.1093 / \mathrm{icb} / 41.6 .1311$

Ikram M, Chibani A, Alemad A, Alkhali A, Belala A, Hadji M, Belghyti D, El Kharrim K. 2016. Etude écologique et entomologique des gîtes larvaires des Culicidés de la Province de Kenitra (Maroc). European Scientific Journal, 12(32): $1857-7431 . \quad$ DOI: 10.19044/esj.v12 n32 p398.

Koumba AA, Koumba CRZ, Mintsa Nguema R, Salako Djogbenou L, Obame Ondo P, Koffivi Ketoh G, Comlan P, M'Batchi B, Mavoungou JF. 2018. Distribution spatiale et saisonnière des gîtes larvaires 
des moustiques dans les espaces agricoles de la zone de Mouila, Gabon. International Journal of Biological and Chemical Sciences, 10(3): 1037-1047. DOI:

https://dx.doi.org/10.4314/ijbcs.v12i4.19

Makanga BK. 2016. Ecologie de la transmission des Plasmodium simiens au Gabon. Thèse de 3ème cycle, Ecole Doctorale Régionale de Franceville, Franceville, p. 191.

Martine Udaga Mungunuti. 2014. Ecologie des larves, élevage et test de sensibilité des moustiques aux différents insecticides usuels en sante publique. Mémoire. Kisangani, RD Congo. 39 p.

Messai N, Berchi S, Boulknafed F, Louadi K. 2010. Inventaire systématique et diversité biologique de Culicidae (Diptera: Nematocera) dans la région de Mila (Algérie). Entomologie Faunistique 63(3) : 203-206.

Mourou JR, Coffinet T, Jarjaval F, Cotteaux C, Pradines E, Godefroy L, Kombila M, Pagès F. 2012. Malaria transmission in Libreville: results of a one-year survey. Malaria Journal, 11 : 1-14. DOI: 10.1186/1475-2875-11-40

PNLP. 2018. Statistique épidémiologique du Niger. Rapport annuel d'activités. Ministère de la Santé Publique, Niamey, p. 40.

Sy O, Konaté L, Ndiaye A, Dia I, Diallo A, Taïrou F, Bâ EL, Gomis JF, Ndiaye JL,
Cissé B, Gaye O, Faye O. 2016. Identification des gîtes larvaires d'anophèles dans les foyers résiduels de faible transmission du paludisme " hotspots » au centre-ouest du Sénégal. Bulletin de la Société de Pathologie Exotique, 109: 31-38. DOI: 10.1007/s13149-016-0469-2

Talipouo A, Ntonga-Akono P, Tagne D, Mbida-Mbida A, Etang J, TchoffoFobasso R, Ekoko W, Binyang J, Dongmo A .2017. Comparative study of Culicidae biodiversity of Manoka island and Youpwe mainland area, Littoral, Cameroon. International Journal of Biosciences, 10(4): 9-18. DOI: http://dx.doi.org/10.12692/ijb/10.4.9-18.

Tia E, Gbalegba NGC, M'bra KR, Kaba A, Boby OAM, Koné M, Chouaibou M, Koné B, Koudou GB. 2016. Etude du niveau de production larvaire d'Anopheles gambiae s.l. (Diptera : Culicidae) dans les différents types de gîtes à Oussou-yaokro au Centre-Ouest et à Korhogo, au Nord (Côte d'Ivoire). Journal of Applied Biosciences, 105: 10170-10182. DOI: http://dx.doi.org/10.4314/jab.v105i1.13.

WHO. 2018. World malaria report. World Health Organization, Switzerland, Geneva, $320 \mathrm{p}$.

WHO. 2018. Rapport annuel. World Health Organization, Niger. 